\title{
Jihel Gée
}

\author{
Astrosophe
}

$(1994,1997,2010)$

\section{"Au Pays de Gilles Vigneault. Jupiter et Pluton - Messagères de l'Indépendance nationale ?'}

\author{
(Le document original tel que publié fut étayé par cinq (5) cartes du ciel)
}

\begin{abstract}
Un document produit en version numérique par Jean-Marie Tremblay, bénévole, professeur de sociologie au Cégep de Chicoutimi

Courriel: jean-marie_tremblay@uqac.ca

Site web pédagogique : http://www.uqac.ca/jmt-sociologue/
\end{abstract}

Dans le cadre de: "Les classiques des sciences sociales" Une bibliothèque numérique fondée et dirigée par Jean-Marie Tremblay, professeur de sociologie au Cégep de Chicoutimi Site web: http://classiques.uqac.ca/

\footnotetext{
Une collection développée en collaboration avec la Bibliothèque

Paul-Émile-Boulet de l'Université du Québec à Chicoutimi

Site web: http://bibliotheque.uqac.ca/
} 


\section{Politique d'utilisation de la bibliothèque des Classiques}

Toute reproduction et rediffusion de nos fichiers est interdite, même avec la mention de leur provenance, sans l'autorisation formelle, écrite, du fondateur des Classiques des sciences sociales, Jean-Marie Tremblay, sociologue.

Les fichiers des Classiques des sciences sociales ne peuvent sans autorisation formelle:

- être hébergés (en fichier ou page web, en totalité ou en partie) sur un serveur autre que celui des Classiques.

- servir de base de travail à un autre fichier modifié ensuite par tout autre moyen (couleur, police, mise en page, extraits, support, etc...),

Les fichiers (.html, .doc, .pdf, .rtf, .jpg, .gif) disponibles sur le site Les Classiques des sciences sociales sont la propriété des Classiques des sciences sociales, un organisme à but non lucratif composé exclusivement de bénévoles.

Ils sont disponibles pour une utilisation intellectuelle et personnelle et, en aucun cas, commerciale. Toute utilisation à des fins commerciales des fichiers sur ce site est strictement interdite et toute rediffusion est également strictement interdite.

\section{L'accès à notre travail est libre et gratuit à tous les utilisateurs. C'est notre} mission.

Jean-Marie Tremblay, sociologue

Fondateur et Président-directeur général,

LES CLASSIQUES DES SCIENCES SOCIALES. 
Cette édition électronique a été réalisée par Jean-Marie Tremblay, bénévole, professeur de sociologie au Cégep de Chicoutimi à partir de :

Jihel Gée

"Au Pays de Gilles Vigneault.

Jupiter et Pluton - Messagères de l'Indépendance nationale ?"

[Article paru dans les revues L'ASTROSPIRALE (Québec) de septembre 1994 et L'ASTROLOGUE (Paris) de juin et septembre 1997 (Nos 118 et 119)]

(Le document original tel que publié fut étayé par cinq (5) cartes du ciel)

[Autorisation formelle accordée par l'auteur le 26 janvier 2010 de diffuser ce texte dans Les Classiques des sciences sociales.]

Courriel : Jihel_Gee@yahoo.com

Police de caractère utilisée :

Pour le texte: Arial.

Édition électronique réalisée avec le traitement de textes Microsoft Word 2008 pour Macintosh.

Mise en page sur papier format : US Letter.

Édition numérique complétée le 4 février 2010 à Chicoutimi, Ville de Saguenay, État du Québec, CND.

\section{Fait avec}

Macintosh 


\section{Au Pays de Gilles Vigneault}

\section{Jupiter et Pluton - Messagères de l'Indépendance nationale ?}

[Article paru dans les revues L'ASTROSPIRALE (Québec) de septembre 1994 et L'ASTROLOGUE (Paris) de juin et septembre $1997\left(\mathrm{~N}^{\mathrm{os}} 118\right.$ et 119)]

(Le document original tel que publié fut étayé par cinq (5) cartes du ciel)

Ce texte - demeuré inchangé hormis léger peaufinage purement stylistique - fut rédigé à l'été de 1994 et initialement publié dans L'Astrospirale, revue québécoise d'astrologie, en septembre de la même année. Le Parti Québécois (indépendantiste) forme le Gouvernement du Québec depuis ce même septembre. Le référendum dont il sera ici question en dernière analyse eut lieu un an plus tard, soit en octobre 1995 [NdA-1996].

«Une fois pris dans l'événement, les hommes ne s'en effraient plus.

Seul l'inconnu épouvante les hommes. »

Antoine de Saint-Exupéry, Terre des hommes

Préliminaire - Bien qu'il s'agisse en ce texte de tenter d'établir objectivement une corrélation significative entre une donnée astrologique spécifique et un mouvement collectif, il reste que l'auteur a choisi délibérément de ne pas jouer «la carte du neutre» sur le plan de ses choix politiques. Cela dit, cet article n'en est pas un de conviction d'idées personnelles, moins encore de propagande : si mes préférences ne sont pas ici dissimulées, en effet, en revanche elles n'interviennent d'aucune manière dans le traitement factuel et épistémologique de l'information. Aussi, qu'il soit bien entendu que j'essaie par la présente - avec toute l'honnêteté intellectuelle propre à un chercheur du comprendre et du mieux-vivre pour les siens de formuler une lecture des astres et non point de les faire «tourner» dans le sens de ma plume. En outre, il va de soi que mes propos n'engagent que moi et que nul ne saurait en être tenu responsable, notamment les éditeurs du périodique qui daignent aimablement publier ici le fruit de ma modeste réflexion. 
Dans une problématique essentielle comme celle du cheminement d'une nation vers sa maturité, il est certainement indéniable - si tant est qu'elle soit elle-même un savoir crédible et digne de ce nom - que l'astrologie est en mesure à cet égard d'éclairer la voie autre que lactée, depuis le reflet des étoiles lointaines, dans l'obscurité silencieuse du coeur des hommes et de la personnalité des peuples.

En regardant la chose d'un peu plus près je pense avoir décelé, et c'est là mon Hypothèse, la prégnance (ou dominance) d'un phénomène astronomique précis : la conjonction longitudinale Jupiter/Pluton (désormais identifiée dans le texte par : C-JP), dont la périodicité est approximativement de 13 années ; laquelle conjonction se voit reconduite, sauf exceptionnelle répétition dans un même signe, dans chacun de ceux-ci consécutivement. L'on pourrait mathématiser le mouvement par la formule : périodicité zodiacale $\mathbf{X}+\mathbf{1}$. Ainsi, la $\mathbf{C}$ se produisit à la fin du Lion en 1955, à la fin de la Vierge en 1968, de même position en Balance en 1981 comme ce le sera identiquement en Scorpion, à la fin de 1994. En revanche, il y a parfois de sérieuses 'anomalies' (explicables par l'orbite fort elliptique de Pluton autour du Soleil), comme l'indique le «pas » d'un seul signe de 1918 à 1943 , alors que la $\mathrm{C}$ prit 25 ans pour faire le trajet depuis le degré 6 en Cancer au $7^{\mathrm{e}} \mathrm{du}$ Lion (période durant laquelle d'ailleurs Pluton fut découverte) - incidemment de la Grande Guerre à la Seconde, entre lesquelles il y eut la belle (?) indolence des années '20. Et du Cancer, peut-être ?

Mon point de départ réside dans la conjonction qui s'est produite - très exactement - dans la nuit du 12 au 13 octobre 1968, plus précisément en début de nuit. C'était la fin de semaine du Congrès de fondation du Parti Québécois, qui eut lieu du 11 au 14. Moment historique s'il en est. Pour la première fois dans l'histoire du Québec (et du Canada d'autrefois, dit français), une force ayant des assises solides dans la population était créée en vue d'une réelle prise en main des destinées du seul État français d'Amérique. Par-delà les événements épisodiques quoique parfois réellement lourds de sens, à l'instar des faits d'arme entourant les Patriotes de 1837-1838, et au delà de strictes revendications d'ordre culturel et linguistique exprimées par de multiples mouvements sociaux, le Québec se donnait clairement un instrument structuré avec l'objectif limpide et avoué de doter la nation québécoise d'un véritable pays.

Une première recherche de corrélations entre le paysage céleste et les événements de cet ordre est demeurée à mes yeux infructueuse. Or lorsque l'on appréhende l'histoire du mouvement indépendantiste contemporain à la lumière de la C-JP (mon argumentation sera donc de type inductif), il semblerait que celle-ci, vraisemblablement, soit l'indicatif d'un véritable moment fondateur. A contrario, et en quelque sorte à titre de contre-épreuve, on constate en effet que la $\mathrm{C}$ ne semble pas avoir eu, dans le passé, une présence significative dans ledit mouvement politique. À la rigueur pourrait-on identifier un prodrome dans la C de 1955 (réitérée en juin 1956, suite à un mouvement rétrograde), si on songe à la naissance, en cascades, à partir de cette période, de plusieurs organisations fermement affichées indépendantistes. Rappelons pour la petite histoire (mais n'y a-t-il jamais de «petite » histoire ?) la création de L'Alliance Laurentienne par Raymond Barbeau en 1957, de L'Action socialiste, fondée par Raoul Roy en 1959 (l'année du «Désormais ! » de Paul Sauvé et de la naissance de la revue Liberté). En parallèle, Marcel Chaput et André d'Allemagne fondaient le Ralliement pour l'Indépendance Nationale (RIN) duquel Pierre Bourgault devint éventuellement le chef. Le chapelet fébrile se poursuit jusqu'en 1964, alors que René Jutras, médecin de Victoriaville, établit les assises du Regroupement National - devenu le Ralliement National en 1966. Toutes ces 
organisations se sont éteintes ou sabordées pour se joindre au Mouvement Souveraineté-Association (MSA), mis sur pied par René Lévesque en 1967 (l'année du «Vive le Québec Libre! » du grand Charles), afin de créer un véritable parti politique dans les heures ponctuelles, et euphoriques, où Jupiter et Pluton convolaient en justes noces au $24^{\mathrm{e}}$ degré de la Vierge.

On pourrait aussi «s'amuser » de conjectures avec le Front de Libération du Québec (FLQ), qui a vu le jour l'année où nos deux planètes entraient en opposition, à mi-chemin de leur périodicité conjonctionnelle. Nous étions en 1963, année qui du même élan enfanta la revue Parti pris, organe de réflexion prônant un Québec fort dans un Canada... ailleurs. Nous étions en pleine Révolution tranquille. «Maîtres chez-nous! », disions-nous fièrement (tout petit garçon, je n'y comprenais goutte mais... déjà fier tout de même). On pourrait certes tenter quelques rapprochements micro-macro / cosmiques sur le climat d'époque. A priori il ne me semble pas cependant que l'on puisse établir de corrélations nettes et concluantes avec les mouvements astraux. Cela reste évidemment à vérifier avec plus de certitude. Un collègue compétent et exercé s’y aventurera peut-être.

En contrepartie, si nous suivons le vecteur temps post-1968, nous constatons que la C-JP ultérieure se produit en 1981: l'année de l'éclatante victoire du parti souverainiste. Un Québécois sur deux plébiscitait le gouvernement en place, et lui offrait les deux tiers des sièges à l'Assemblée Nationale. Jamais ne s'était vue dans notre courte histoire une confiance populaire d'une telle ampleur pour une force indépendantiste. Par la grande lunette de l'Histoire, il faut également préciser que si la naissance du PQ fut pour la nation un acte que je qualifierais sinon de fondateur, de substructurel, il faut dire en revanche que le report au pouvoir du PQ révélait à l'envi que le (premier) mandat électoral de 1976 ne pouvait plus être interprété comme un « accident de l'Histoire », ainsi que d'aucuns ont tenté de le présenter à l'époque. D'ailleurs, et notamment pour cet aspect « soutènement de l'acquis », j'estime personnellement que cette élection constitue à ce jour, sur le plan historique, la crête du mouvement d'Indépendance québécois. Ce qui au demeurant semble se voir corroboré par l'hypothèse de la C-JP, laquelle en effet préféra «parrainer » 1981 en lieu et place de 1976.

Sauf que... Sauf qu'il y eut, bien sûr, le cuisant - et non moins historique - revers de 1980. Une véritable «Première » dans l'histoire des peuples : se refuser à soi-même l'Indépendance! Quelque chose comme un grand'enfant (attardé ?) de 37 ans, qui se menotte à la patte d'un fauteuil du salon de papa et maman - effrayé à l'idée d'aller travailler tous les matins pour se construire une «personnalité », et de devoir faire sa lessive tout seul... On me fera remarquer, non sans à propos, que le gouvernement qui fut élu en 1981 avait mis en veilleuse, suite à 1980, la raison même de son existence - «Un pays à bâtir»-, et que dès lors il est excessif de ma part d'annoncer un grand moment de Liberté, comme je le sous-entends à l'instant. Or je répondrai que c'est l'Histoire qui... répondra.

Je ne sais si, au référendum probable de 1995, nous préférerons nous ouvrir définitivement les veines ou bien nous ouvrir enfin à nous-mêmes (sans la culpabilité d'estimer que par là même nous nous fermons à l' «Autre ») ; mais si cette dernière éventualité de l'alternative devait se confirmer, je crois qu'on sera amené, rétrospectivement, à interpréter l'élection de 1981 comme le mortier de ce qui allait survenir. L'Histoire a parfois de ces langueurs, de ces chants de sirène comme pour se mieux faire désirer. Lorsque René Lévesque nous dit : «À la prochaine fois ! », un soir de mai '80, un jour où il n'était certainement pas très fier d'être Québécois, peut-être avait-il l'intuition du mouvement... 
cyclique des planètes, ou avait-il conservé les engrammes d'une vieille lecture philosophique. C'est le grand penseur allemand Hegel qui écrivait, en effet, dans ses Vorlesungen über die Philosophie der Geschichte, qu' :

«Une révolution politique est, en général, sanctionnée par l'opinion des hommes quand elle se renouvelle. C'est ainsi que Napoléon succomba deux fois et que l'on a chassé deux fois les Bourbons. La répétition réalise et confirme ce qui au début paraissait seulement contingent et possible. » [Hegel renvoie ici à Louis XVI en 1793 et Charles X en 1830, puis à Napoléon qui, de 1814 à 1815, passa de l'île d'Elbe à Sainte-Hélène... par Waterloo]

Après 1968 et 1981, assisterons-nous en 1994-1995 à une possession entière de notre destin collectif ? Et trois fois plutôt qu'une - ou deux. Ou confirmerons-nous 1980 ? Nous jetterons un coup d'oeil làdessus à l'instant. Pour le moment, penchons-nous sur les thèmes astraux de 1968 et de 1981.

\section{Carte A - 13 octobre 1968 : Naissance du Parti Québécois}

Un survol rapide (j'exclus les aspects mineurs) indique à mes yeux une formidable impulsion, originelle, par Uranus d'abord qui forme une triple $\mathrm{C}$ avec $\mathrm{J}$ et $\mathrm{P}$ ( $7^{\circ} \mathrm{d}$ 'orbe), mais également avec une $4^{\mathrm{e}}$ source, Mars, qui alors à $10^{\circ}$ d'orbe s'en vient bientôt rejoindre le groupe. Uranus, Pluton et Mars se rencontrant chez Jupiter : c'est du plutonium dans la tête que cet agrégat. Ou un commando ! Ah ! ce que l'on a dû discuter ferme cette fin de semaine-là. Toutefois, il semble que l'on maîtrise bien la démesure potentielle de cette 'nitro'. C'est que les doux aspects de Neptune et de Vénus canalisent intelligemment la poudrière. Même Mars ne résiste pas aux charmes de la Lune, qui lui tend un long sourire de $60^{\circ}-$ en sex[e]til. Ajoutons que le tout est situé en terrain sûr. Ce n'est certes pas dans la Vierge méthodique et réfléchie que l'on risque de poser le pied sur une mine (le gros bon sens rusé de René Lévesque... ? dont c'est au surplus le signe solaire...).

On constate - donnée tout à fait remarquable - que la carte n'indique qu'un seul aspect dissonant, en l'occurrence l'opposition de Saturne à la C Mercure/Soleil, laquelle à mon avis n'a pas d'incidence affirmée sur le noyau JP, quoique Mercure soit maître de celui-ci par la Vierge. Il demeure que ce trait révèle surtout un solide jugement, qualité précieuse entre toutes. En conséquence: aspect de tempérance sinon de sagesse, et en outre devant l'énergie solaire toujours incandescente. Voilà un exemple type illustrant, pour ainsi dire par antithèse, la méprise entourant l'appellation, parce que trop souvent 'incontrôlée', du soi-disant aspect dissonant.

Toutefois, symétriquement, on verra plus loin que cette présence de Saturne n'était sans doute pas fortuite : on doit savoir en effet que l'excès de... tempérance, qui est l'inhibition, reste un excès tout de même. Vestige sans doute du vieux catholicisme baignant encore les gènes d'une famille inquiète, et qui a conservé le réflexe frileux des peuples colonisés (l'image la plus éclatante à cet égard m'apparaît être la remise des 'clés' de la nation - et à quatre reprises s.v.p.! - à un individu de la stature d'un Robert Bourassa). C'est encore Saint-Ex. qui écrivait que «l'esclave fait son orgueil de la braise du maître... ». Il est vrai, hélas, qu'un peuple sans ambition ne craint pas les échecs.

Mais trêve de circonvolutions qui, pour l'heure, empiètent sur l'avenir. Il faut dire haut que cette domification du 13 octobre '68 nous signale une superbe énergie, quelque chose d'analogue à une 
passion comme il y en a peu : puissante, gourmande mais équilibrée. Je crois qu'on assistait alors moins à un acte strictement politique que lucidement amoureux. Faire acte d'amour de soi, c'est le premier pas vers un authentique amour de l'autre, et peut-être surtout la condition d'acceptation d'être soi-même aimé par l'autre. Dans un hommage à Gilles Vigneault, l'ex-député - mais à tout jamais poète - Gérald Godin disait que le pays du Québec apparaîtra «quand il sera suffisamment aimé ». C'est exactement ce que je lis dans cette carte du ciel. Mais l'est-il, le sera-t-il jamais suffisamment ? L'astrologue cherche, le citoyen maugrée. Il y a carré entre les deux demi-cercles de mon cerveau.

\section{Carte B - Victoire du PQ aux élections du 13 avril 1981}

Jupiter et Pluton ne se rencontrent au même degré que plus tard à l'automne. À la date de l'élection, les deux astres ne sont pas en conjonction. Les accointances longitudinales rigoureusement exactes (presque «magiques » et à marquer d'une pierre blanche) - à l'instar de la conjonction survenue dans la nuit du 12 au 13 octobre 1968 - sont extrêmement rares en astrologie ; d'où leur valeur ajoutée, si on me permet l'expression. Ici encore, nous avons un thème magnifique (et ce n'est pas le 'partisan' qui s'exclame). Le triple Grand trigone (!) formé par les aspects de Neptune et de la Lune avec la triple conjonction Soleil, Vénus et Mars est un phénomène tout à fait exceptionnel. Ce qui à nouveau détone a priori, c'est le caractère affectif de la toile fabriquée par ce lignage d'aspects. Le 'Feu' du Soleil, partie prenante au triangle (exalté en Bélier et maître de l'un des angles du trigone par la présence de la Lune en Lion) stimule puissamment vers l'action - autre élément franchement déterminant - la triplicité de Feu (Sagittaire, Bélier, Lion). Or comme si ce n'était suffisant, Mars impliqué et dans la $\mathrm{C}$ et dans le trigone est son propre maître par sa position dans ce Bélier, alors que Vénus intensifie son opposition à Pluton par son exil dans le même signe. Nous avons là une énergie absolument phénoménale quoique fermement contrôlée - au sens de canalisée, et par distinction de contrainte. Un brasier, vous dis-je.

Décidément, on assiste à un véritable acte d'amour. C'est presque indécent que de procéder à l'érection d'un pareil thème. Il est clair cependant que cette passion ne compte pas rester 'au lit' ...toute la journée. C'est une puissante émotion qui clame fort : «J'ai le goût de construire! » Au surplus (mais est-ce possible ?!?), cette énergie se voit carrément surmultipliée par Pluton qui oppose résistance (nécessaire à la création: les premiers de classe à la petite école deviennent rarement, faute d'adversité, de grandes personnalités) à la triple conjonction en Bélier (dont il serait Ouf ! - l'un des maîtres, selon une certaine tradition astrologique), mais pour la 'réverbérer' à nouveau - tel un monumental circuit électrique fonctionnant à plein régime - dans la même triplicité de Feu par le double sextil simultané à Neptune et à la Lune.

Nous ne sommes pas ici devant une formulation de type : «Tu es belle, je suis beau, contemplonsnous. » Cette signature planétaire nous dirait plutôt: "Vous êtes beaux, alors créez la beauté tout autour de vous. » C'est le «Regarder ensemble dans la même direction » de notre 'pilote de guerre' de prédilection. Cela entendu, il faut bien avouer qu'il est fort étonnant d'apercevoir un tel "goût de bâtir » dans un événement électif comme celui-là, alors que les mêmes protagonistes se faisaient littéralement lessiver moins d'un an auparavant, à la faveur du référendum. Naïveté ou confiance dans l'avenir? 
On constatera d'autre part qu'Uranus continue, comme en 1968, d'offrir son soutien à Jupiter, lié ici à Saturne (exalté en Balance) qui a, de mon avis, un rôle également de soutien bien plus que de frein. Saturne et Jupiter ne sont pas toujours du même avis, loin s'en faut. Mais ce sont deux adultes qui se respectent, capables de nobles gestes, et dont la réflexion du premier est nécessaire à la bonne marche des grands projets du second - et réciproquement. Deux individus qui ne s'aiment pas profusément, certes ; mais qui préféreraient perdre une main plutôt que de trahir leurs engagements l'un envers l'autre. Une conversation Jupiter / Saturne, c'est en quelque sorte une perle de civilisation (et pas uniquement de civilité).

En corollaire sur ce thème de 1981, un mot sur la régularité progressive de la position de la Lune dans la fameuse triplicité. Composante du dossier qui peut être interprétée contradictoirement; puisque sauf exception l'on ne retient habituellement que les planètes dites lentes dans l'analyse des mouvements collectifs. À cet égard, et a contrario, donc, si l'on ignorait la Lune dans notre interprétation, celle-ci se révélerait beaucoup moins étoffée et par là d'autant moins convaincante. Aussi compte tenu que la C-JP de 1981 n'était pas encore formellement établie au printemps, et que d'autre part la formidable triplicité de feu, en concomitance, se voyait réduite au simple (quoique important tout de même) trigone de la triple C avec Neptune en Sagittaire, il s'ensuit que l'examen qui précède se fût révélé hautement problématique si je n'eus fait référence, à l'encontre du paradigme en vigueur, à la plus véloce des «planètes ».

En revanche, si l'on retient, précisément, la ponctualité inhérente à un événement sociétal nettement circonscrit dans le temps, donc dans l'Histoire, l'élection d'un gouvernement en l'occasion, la présence de l'astre le plus rapide à un degré tout à fait stratégique du thème confère à celui-ci au contraire - une signification inattendue et remarquable. De fait, si l'événement analysé s'était produit ne serait-ce que 24 heures plus tôt, mes conclusions se seraient avérées largement moins pertinentes, sinon carrément déconnectées. Or c'est bel et bien le 13 avril que survint ladite élection. De plus, la triplicité devient effectivement plus puissante en terme d'exactitude tout au long de la journée. Le thème ayant été domifié pour 07:00, les aspects de la Lune $\left(18^{\circ}\right)$ s'appliquent en effet avec grande précision sur Mars $\left(21^{\circ}\right)$ puis Soleil $\left(24^{\circ}\right)$ dans le courant du jour, et enfin sur Vénus et Neptune $\left(25^{\circ}\right)$ en début de soirée. Vers 20-21 heures, nous avions un Grand trigone de feu parfait à $25^{\circ}$ impliquant Neptune, Vénus (à un seul degré du Soleil) et la Lune, respectivement en Sagittaire, Bélier et Lion: exactement au moment où Radio-Canada annonçait officiellement l'élection majoritaire du PQ... Il serait extrêmement difficile de trouver une signature astrale plus personnalisée que celle-là. Si j'avais l'esprit un peu moins saturnien, je parlerais d'emblée d'un rayon d'amour, voire, d'un orgasme littéralement cosmique. Décidément, les dieux ont dormi au Québec cette nuit du 13 avril. Et ils s'y sont bien amusés.

En conséquence j'estime que l'argumentation, à l'effet de s'opposer à l'utilisation de la Lune dans le décodage de l'événement de ce jour, se retourne contre elle-même. C'est au contraire la Lune qui marque très profondément la domification de ce thème. Elle y interprète un rôle tout à fait crucial. Sans (presque) l'ombre d'un doute, et sans fausse modestie, je dirais : CQFD. Et tant pis pour mes scrupules de chercheur, sceptique comme par définition. Au demeurant, est-il utile de rappeler la symbolique sociale de la Lune ? Je cite le (sérieux) Traité d'astrologie d'André Barbault : «Lune : la foule, le peuple, le public, la nation, la république ». J'arrête ici. Je vertiginise. 
Dernier élément enfin, banal en apparence mais probablement annonciateur de difficultés sinon d'événements funestes. Devant cette carte extraordinaire, a priori, on se demanderait ce qui a bien pu se produire pour que l'on aboutisse finalement au Québec médiocre et 'provincial' actuel. Je le vois dans le mouvement rétrograde de toutes les planètes lourdes, de Jupiter à Pluton! (par opposition, notons au passage qu'en 1968 seule Saturne 'avançait en arrière'). Sans tomber dans la mesquinerie ou les arguments ad hominem, il faut dire que les Trudeau, les Chrétien et les Bourassa pouvaient certainement trouver là un formidable creuset pour retenir le "grand'garçon" à la patte du fauteuil... Ce thème dévoile à mes yeux le gaspillage, je dirais éhonté, de la promesse d'un avenir incomparable. C'est un peu donner naissance à un Léonard de Vinci et, du moins au Québec, l'installer devant Télévision Quatre-Saisons (ou american Rock-détente ?) en l'empiffrant de Rambo, de croustilles et de coca-cola. Nous n'avons pas été dignes du cadeau des dieux. Et ceux-ci pourraient nous le faire payer très cher : l'Histoire se montre rarement généreuse.

\section{Objectif : un Pays nommé QuÉBEC}

Si l'hypothèse d'associer la C-JP au mouvement d'Indépendance tient le coup, que peut-on, dans ce cadre, appréhender d'un éventuel référendum ? D'abord, il est clair que celui-ci devra se tenir, à mon avis, au plus tard en août 1995 ; alors que la C s'estompera par delà les $10^{\circ}$ d'orbe à compter de cette date. D'autre part, c'est autour du 2 décembre 1994, donc moins de trois mois après l'élection du 12 septembre, que la $\mathrm{C}$ sera la plus 'pure', à $28^{\circ}$ exactement du Scorpion. Si l'équipe gouvernementale souverainiste, advenant bien sûr son élection, devait reporter l'événement au-delà de décembre, c'est en juillet que le moment m'apparaîtrait le plus propice en terme de concision d'aspect, bien que l'on ne puisse retrouver alors la «perfection astrométrique » du 2 décembre.

Cela dit, sur le plan politique il serait douteux que l'on opte pour un appel aux urnes en pleine canicule (autre nom pour l'étoile Sirius). Au reste, l'on sait que la minutie de l'aspect n'est pas en soi un gage de succès. On l'a vu notamment en 1981. Enfin, il n'est pas impossible que cette précise C-JP de décembre soulignât plutôt l'élection en cours, étape préalable nécessaire, et par là incontournable, au référendum sur l'Indépendance. Il faudrait clairement comprendre alors élection/référendum sous un même mouvement astrologique, à quelques mois d'intervalle près. Or ce semble tout à fait la stratégie du Parti Québécois. Mais quoi qu'il en soit de ces considérations logistiques, nous devons tout de même poser l'éventualité du référendum aux deux quantièmes retenus; et cela en conservant à l'esprit que le commentaire portant sur l'hypothèse de décembre pourrait aussi bien s'appliquer au résultat de l'actuelle campagne électorale (août 1994).

Ainsi, deux périodes sont à envisager : le début de décembre 1994 (et ce malgré les sondages actuels, qui n'interviennent pas dans mon analyse) ou le plus tard possible en juin 1995 (le 24 juin 1995 de M. Jacques Parizeau serait-il prophétique ?), alors que l'orbe sera inférieur à $10^{\circ}$ après le 18 juin. Nous allons voir qu'il ne m'est pas possible ici de sortir un lapin de mon chapeau. Les deux thèses, décembre ou juin, se défendent bien respectivement, surtout la seconde. Ce qui est indubitable, c'est qu'une victoire référendaire ne surviendra pas... en regardant le ciel. Ce ne sont pas les astres qui produisent ou empêchent les désastres. Cela est certain. Ce sont les Hommes qui sont ou ne sont pas à l'écoute d'eux-mêmes. Car le ciel symbolique est à ceux-ci ce que sont les étoiles et la Lune au Soleil : un simple miroir de Soi. Point de force, mais une forme. Qui informe. 


\section{Carte C - Référendum : Hypothèse du 2 décembre 1994}

La C-JP se produit en Scorpion dont Pluton est expressément le maître. Au départ, je vois donc un grand potentiel de 'renversement'. Il y a là une volonté et une capacité réelle de rompre un vieux cordon ombilical devenu non seulement inutile, mais dangereux. Et si Lune et Mercure s'unissent à JP pour donner une quadruple C, tout en en formant une autre avec le Soleil plus loin en Sagittaire, il reste que ce bassin d'énergie ne doit compter que sur lui-même. En effet, sauf un timide sextil depuis Uranus, rien de l' "extérieur" ne viendra donner un coup de main à la mouvance indépendantiste. La force est tout de même fort impressionnante. Les 5 planètes en C (si on inclut le Soleil, plus éloigné à $12^{\circ}$ d'orbe de JP) sont maîtrisées ou par Jupiter (Sagittaire), ou par Pluton (Scorpion) - soit les deux planètes déterminantes dans notre étude.

Nous avons donc là, de $28^{\circ}$ en Scorpion au degré dixième du Sagittaire, une formidable puissance de changement. Sans compter que les deux signes sont des signes de haute indépendance, capables de croire en eux-mêmes. Lune et Mercure exactement au même degré laissent prévoir une argumentation qui s'adressera à l'intelligence des gens. La C Lune / Soleil pourrait également laisser planer la possibilité que l'électorat ralliera cette même raison à son émotion, ce qui est l'illustration de l'équilibre psychologique (et social du même coup). Peut-être est-ce là le signal que la collectivité ne nécessite plus le port et de la ceinture et des bretelles (entendez: Pierre Elliott Trudeau et René Lévesque, ou... Lucien Bouchard et Robert Bourassa/Daniel Johnson). Lune et Mercure conjoints à Soleil, ce peut être fort bien la raison qui rejoint le coeur : un adolescent qui devient adulte.

Pourtant, ce n'est pas dans le sac. Les deux gants de boxe planétaires, Mars et Saturne, se donnent la main (dans le gant) pour contrer ce puissant mouvement. Tel un laser en miroirs, leur opposition l'un à l'autre canalise leurs énergies respectives pour rasseoir la fameuse C. Ce qui est remarquable ici, c'est que nous assistons à l'union affirmée de forces par nature dissemblables, voire incompatibles : la marsienne, fonçeuse, revendicatrice et vindicative, puis la saturnienne, lente, ombrageuse et résistive, sinon rétive. C'est le taureau des corridas et le bœuf de la ferme, le coq des combats et le chapon renfrogné. Leur disposition, l'une en Lion, l'autre en Poissons, éclaire à mon sens le phénomène. Il m'est difficile de ne pas voir en cela la combinaison de l'atavisme, d'une part, et de la puissance ferme et calculée qui a les moyens de sa négation, d'autre part.

En d'autres termes, il s'agit de l'alliance contre-nature (mais pas vraiment, au fond, si on regarde l'Histoire, alors que ces pouvoirs s'avèrent assez souvent des alliés objectifs; et ce, bien que l'un, toujours le même, devienne non moins fréquemment l' 'instrument' de l'autre) entre la crainte du changement, qui baigne pour ainsi dire naturellement les populations, et le refus systématique de modification au statu quo ante propre à de grands intérêts, privés ou corporatifs, qui font leur beurre de cette stagnation sécurisante, les pouvoirs financiers en particulier (comme si, rétroactivement, le 'calme' du maintenant n'était pas le résultat du renversement, parfois brutal qui plus est, des façons d'hier). La grande distinction entre les deux forces, c'est que l'une peut «dérégler la machine à progrès » par la force d'inertie et la pusillanimité : c'est moins être contre que de «ne pas être pour ». C'est laisser aller plutôt que d'affirmer. Bref, laisser décider les autres. Il y a même des premiers ministres comme cela! De l'autre côté, on identifie une force 'activement contre', et dont la puissance d'obstruction s'évalue souvent en dollars. 
Le Saturne réfractaire conserve une forte emprise sur la foule (Poissons). Mars est puissant et articulé, voire imbu (Lion). On assiste définitivement à un combat de titans contre C-PJ en Scorpion, lui-même fier et convaincu. Quelle en sera l'issue, dans l'hypothèse d'un verdict arrêté ce 2 décembre ? Bien malin ou présomptueux celui qui répondra avant l'échéance démocratique. Je crois tout de même que l'atavisme populaire se verra nettement moins prononcé qu'autrefois. En effet, Saturne n'attaque pas directement C-JP, mais plutôt ses compagnons de route immédiats, Soleil, Lune et Mercure, à mon avis planètes moins cruciales dans le portrait du jour. Saturne n'est pas très puissante par ellemême non plus, sa position en Poissons la rendant presque déficiente. Elle maîtrise certes Uranus et Neptune, mais ces dernières n'ont pas d'incidence contrariante ou contraignante marquée sur l'enjeu. Au surplus, c'est Jupiter qui est son maître.

J'en conclus que la masse populaire (expression que j'utilise ici non par élitisme mais en référence à la nature Poissons, avec sa consonance [parfois] un peu molle et malléable) n'est pas rebelle de façon ferme au projet. De bons arguments sauraient la convaincre de la beauté et de la grandeur de l'entreprise proposée. Les forces antagonistes clairement affichées telles, quant à elles, ne changeront pas d'opinion en chemin, assurément. Mais je ne vois pas en dernière analyse comment un Mars, aussi athlétique soit-il (et ce, même s'il domine également le Scorpion avec sa soeur, pour l'heure ennemie ponctuelle : Pluton), pourrait renverser la vapeur du couple Jupiter/Pluton chaussé des espadrilles d'un Mercure en plein Soleil, et de surcroît sous l'oeil approbateur de l'astre féminin de la nuit (Lune : le peuple). Autre élément exceptionnel : il n'y a aucune, je dis bien aucune planète rétrograde dans ce thème. L'enfant, malgré une gestation difficile, naîtra-t-il naturellement parce que le temps est tout simplement venu?

En définitive, je dirais que la population est ouverte à l'idée d'Indépendance, mais qu'elle ne consentira pas au projet sans être longuement et patiemment courtisée. Elle se laissera désirer jusqu'au dernier instant. En corollaire, et pour ainsi dire de façon stratégique, les forces souverainistes auraient grand avantage à travailler le maillon de l'opposition Mars/Lion - Saturne/Poissons, c'est-àdire le discours que tiendront le grand capital et les fédéraux aux Québécois. Je veux dire en cela que les millions et les infamies de dessous de table ne seront pas suffisants cette fois-ci (en regard à 1980) pour faire échec à la Liberté. Le va-tout se jouera dans la proximité entre ces gens-là, comme dirait Brel, et l'électorat. En termes concis, la nation ne se vendra pas au plus offrant, mais s'offrira cependant au plus convaincant. Alors, souverainistes, à vos plumes et à vos écrans. Un (seul) Bourgault, ce n'est pas suffisant !

\section{Carte D / Carte E - Référendum : Hypothèse des 23 ou 29 juin 1995}

L'examen se concentre sur le 23. Sans doute ai-je tendance à croire que l'éventuel gouvernement préférera le momentum pré-fête nationale. Peut-être à tort. De part et d'autre. Les deux thèmes sont identiques sur le fond, sauf que la Lune me semble promettre l'instillation d'une fraîche énergie, vivifiante et apaisante tout en un, le 29, alors que le satellite de la Terre se combinera aux autres dimensions décrites ci-contre pour offrir un Grand trigone d'eau avec Saturne et Pluton, depuis son royaume en Cancer. J'incline à penser qu'il y aura un «je ne sais quoi » dans l'air ce jour-là, lequel pourrait le cas échéant moduler un doute en audace. Et surtout un goût de partage et de 
communication, sinon de communion. Quelque chose comme un geste de l'âme, apte à faire un instant oublier l'hypothèque ou les traites sur l'auto, et à nous rappeler que nous sommes peut-être autre chose qu'un estomac et un sac d'hormones.

Au départ, la C-PJ se voit moins puissante que dans le thème précédent. En contrepartie, et c'est effectivement très important, Pluton et Jupiter se retrouvent dans leur domicile respectif. C'est une situation de conjonction qui ne se reproduira pas avant... au moins 250 ans, et plus sûrement un demimillénaire - voire plus ! (La révolution de Pluton atteint le quart de millénaire). Pluton est en Scorpion, Jupiter est en Sagittaire. Chacun peut ouvrir totalement ses ailes, étant «chez lui », et en même temps ils peuvent s'unir dans un projet commun. Un vrai couple moderne, quoi. Je suis bien obligé encore une fois de dire - et faute de me répéter, quoique pour des motifs différents de ceux évoqués dans les explorations antérieures - que c'est encore là un phénomène astral exceptionnel.

Je ne suis pas loin de penser d'ailleurs que le grand orbe de presque $10^{\circ}$ de la C-JP (à quelques minutes spatiales près) constitue une dimension plutôt créatrice que débilitante de l'aspect. Ils peuvent tous les deux à la fois s'émanciper et s'épauler : émonder, nettoyer, défricher et construire presque du même mouvement. C'est en quelque sorte une Révolution française immédiatement mise au service de la patrie par l'énergie fougueuse d'un Napoléon, mais sans devoir en passer par la terreur de 1792-1794. Fascinant. Je dirais, dans nos mots d'ici : une véritable Révolution tranquille II. Vraiment, en effet, une très grande force et très tranquille. Ce qui au demeurant est d'ores et déjà constitutif de cette fameuse conjonction, de 1968, voire 1955, jusqu'à ce jour: un ordre de changement immédiatement créateur. Je crois que nous n'avons pas idée de la beauté du peuple que nous pourrions être. Ce qui incidemment n'a rien à voir avec le chauvinisme, qui serait tout au contraire l'éloge de la petitesse, le confort de la mesquinerie et le mépris de la grandeur - laquelle, à l'instar du franc regard d'un enfant, nous est encore insupportable."

L'autre facteur qui retient tout de suite mon attention, c'est (encore) Saturne. Des cinq cartes du ciel retenues, non seulement c'est la seule où celui-ci n'oriente aucun dard sur qui que ce soit, mais qui plus est offre un solide trigone à Pluton lui-même, en plus de relayer ses bonnes dispositions par le truchement du double sextil de la conjonction Uranus/Neptune en Capricorne. Nous avons là réunies les quatre planètes les plus lentes du système solaire, ce qui imprègne fortement les décisions collectives du moment, rehaussées au surplus par la cinquième, qui est bien sûr Jupiter, branchée sur les faisceaux d'énergie de ces premières par sa conjonction avec Pluton. On perçoit donc nettement ce que j'appelle des tendances lourdes en affinité avec ladite conjonction - noyau euristique de notre argumentation. De plus, on remarquera que Jupiter est, avec Neptune, maître de Saturne : tous les deux, nous venons de le dire, en termes amicaux avec Pluton. Enfin, il n'est pas inutile, certes non, de mentionner que dans les cinq (5) domifications la planète Neptune (= collectivité / et sauf une

\footnotetext{
À tout hasard de l'intérêt du lecteur, signalons que dans une livraison printanière de l'an 2000 de la revue L'Action nationale, quelques années plus tard, et parmi d'autres prestations analogues du même, l'auteur est revenu sur ce « thème » politique à la sauce québécoise dans un court texte intitulé : «Qui sort, digne ! Franchir le miroir de notre schizophrénie collective ». [NdÉ-2010]
} 
opposition 'douce' à Lune en carte «E ») se révèle bienveillante en permanence, Uranus [= Personnification même de l'Indépendance] la suivant de très près à cet égard. Voilà qui appuie ou corrobore les lames de fond, les tendances historiques.

Les forces explicitement antagoniques ne font pas le poids. Ce sont plutôt les mouvements rétrogrades qui me préoccupent: toujours cette sempiternelle force d'inertie qui dit non en ne disant rien. Décidément, l'Indépendance ne fait plus l'objet d'attaques en règle : elle n'a plus à se "défendre" ou à se justifier comme une réprouvée stigmatisée sur la place publique. Mais elle ne convainc pas illico pour autant. Il faudra réellement un travail de terrain. L'adversaire n'est plus ce cruel chevalier noir armé jusqu'aux dents. L'ennemi porte un nom nouveau : Soporifie.

En ce qui concerne les éléments réfractaires actifs, on constate que Jupiter se moque littéralement, en ses terres, des hauts cris de Mars qui tente de s'allier Mercure et Vénus. On notera en outre que les deux seules sources d'opposition en carte « $\mathrm{D} »$ proviennent des domiciles de Mercure, soit Vierge et Gémeaux. Si en décembre les forces d'opposition se révélaient somme toute traditionnelles l'atavisme plébéien d'une part, les profiteurs de l'immobilisme d'autre part - il est inusité de constater que les plus fortes critiques pourraient ici provenir des milieux intellectuels. Ce qui a priori serait tout à fait invraisemblable, compte tenu que ce sont justement les intellectuels qui, depuis plus de trente ans, ont à toutes fins utiles porté le flambeau de l'Indépendance au Québec.

Mon hypothèse à cet égard est que les véritables intellectuels (c'est-à-dire les individus qui réfléchissent, non ceux qui se mettent au goût du jour et du prêt-à-penser) se sont tus - et notamment depuis 1980. On sait d'ailleurs pourquoi. Au demeurant, aucune planète de réelle méditation n'est ici impliquée. Mars joint à Mercure (trônant en Gémeaux) donne sans doute de la 'gueule' et Vénus de la séduction. Mais c'est éphémère comme un condom que tout cela. Du dandysme. Et ce, même si Mars a un droit de regard sur le logis de Pluton.

Je retiens en particulier cette pensée molle d'intellectuels de bistrots, qui pose la suprématie d'un monde sans frontières où le 'nationalisme' ferait figure d'archaïsme. C'est-à-dire ce type de pensée qui engendre lui-même les excès parce que elle-même devenue incapable de rigueur, confondant allègrement fermeté d'opinion et fermeture d'esprit. C'est l'indolence du Grand-Tout. Mais qu'est-ce que ce Grand-Tout actuellement sinon la sous-culture, la violence et le dollar étatsuniens ? Voire, la tyrannie d'une langue unique laminant «la différence » partout sur son passage. Associer une volonté de Liberté et de responsabilité collective, comme c'est le cas au Québec, par exemple, à des horreurs ultra-nationalistes, a fortiori sanguinaires, relève de la bêtise, du ridicule (ou de l'extrême mauvaise foi) dans le meilleur des cas. Aussi bien affirmer, à ce compte-là, que tous les amants de la musique beethovenienne sont des barbares sous motif qu'Adolf Hitler et Hermann Goering se délectaient sans 'mesure' des compositions du fameux Viennois d'adoption.

C'est à mon avis de la fantasmagorie d'adolescent (et ici le citoyen philosophant prend le relais du chercheur-astrologue) d'affirmer que les frontières sont par définition des murs. Ce sont plutôt des fenêtres ouvertes à tous les possibles, y compris, quand nécessaire, à celui de devoir être closes comme on le fait parfois devant un écran de télévision devenu tout à coup imbécile par surdose de sang, d'armes à feu, d'automobiles, de chair rarement chérie, et bien sûr de français approximatif appuyé sur la canne de l'anglaisement. Je ne résiste pas à citer de nouveau Hegel, celui de la Logik 
cette fois : «Le concept intégratif de toutes les réalités (der Inbegriff aller Realitäten), quand elles sont pensées sans limite, parvient au néant vide ».

Nul n'est de nulle part. Nous sommes tous d'un lieu et d'une époque. Le monde réside avant tout au fond d'un Moi assumé, non dans un Moi contourné, évité, fui et/ou difforme et malbâti : il en est de même des nations. Si je ne suis plus personne, il me devient impossible d'être-avec-tous : dans un «Je t'aime », il y a un 'je' qui n'est pas moins prégnant que le «t'aime ». Il n'y a pas d'amour, il n'y a que des amoureux ; avec leurs "frontières" respectives, à commencer par leur personnalité et leur corps. Et dieu ! qu'elle est belle la frontière d'un individu avec 'son' intelligence. Et dieu ! qu'elle est belle la frontière du corps de la femme aimée ! Aimer - soi-disant - l'humanité constitue trop souvent le meilleur alibi pour n'aimer personne. Voire, la caution de tous les excès.

Mais place à l'action désormais.

Et puissions-nous ensemble, citoyens, avoir le courage de la Liberté. 